\title{
Molecular dynamics simulations of dendrimer-encapsulated $\alpha$-Keggin ions in trichloromethane solution
}

\section{- Supporting Information-}

Ralf Brodbeck, Thorsten Tönsing, Dirk Andrae ${ }^{1}$

Theoretische Chemie, Fakultät für Chemie, Universität Bielefeld

Postfach 1001 31, D-33501 Bielefeld, Germany \{ralf.brodbeck1, thorsten.toensing, dirk.andrae\}

Quni-bielefeld.de

Dirk Volkmer

Institut für Anorganische Chemie II, Universität Ulm

Albert-Einstein-Allee 11, D-89081 Ulm, Germany

dirk.volkmer@uni-ulm.de

January 28, 2008

${ }^{1}$ Corresponding author. 


\section{A Supplement}

\section{A.1 Computational details}

All Kohn-Sham density functional calculations for polyoxometalates (POM) and dendrimer ions were performed with the GAUSSIAN03 program package, ${ }^{1}$ using the gradient-corrected B3LYP hybrid density functional ${ }^{2,3}$ (with the VWN-III, not the VWN-V functional). Effective core potentials (ECP) and associated spherical Gauss-type basis sets of Stevens and coworkers $^{4,5}$ were used for all centres (no ECP, only basis set, for hydrogen). For the polyoxometalates, polarization and diffuse functions (see Table 1 for their exponents) were added, this basis set is termed $\mathrm{SBKJC}+(d, f)$. The basis sets for the dendrimers were augmented by polarization functions (taken from the GAUSSSIAN basis set database) on all non-hydrogen centres, this basis is termed $\operatorname{SBKJC}(d)$. Incremental buildup of the Fock matrix was observed to cause convergence problems for the POMs, and therefore was disabled. For the structure optimizations we used an integration grid of 99 radial shells with 590 angular points per shell for all atoms (pruned for nonmetals, unpruned for metals). The threshold for maximum force was $4.5 \cdot 10^{-4}$ a.u., the threshold for maximum displacement in internal coordinates was $1.8 \cdot 10^{-3}$ a.u. This lead to equilibrium structures with energies consistent to $10^{-7}$ a.u. (the same accuracy as for the electronic energy).

State-averaged MCSCF calculations (using the $\operatorname{SBKJC}+(d, f)$ basis set) for the states $5 d^{4} 6 s^{2}{ }^{5} D, 5 d^{5} 6 s^{1}{ }^{7} S$, and $5 d^{6}{ }^{5} D$ of the tungsten atom were performed with the GAMESS program. ${ }^{6}$ From the electron densities of these states, radii of density isovalue surfaces with $\rho=0.002$ a.u. were obtained. The arithmetic mean of these radii was taken as the LennardJones parameter $\sigma_{i i}$ for the tungsten atoms. 
Table 1: Exponents $\alpha_{l}$ for diffuse and polarization Gauss-type basis functions. The exponents were obtained via exponent scaling (method a), from the Gaussian03 basis set database (b), or by maximization of atomic correlation energy (c) (Zn: MP2/SBKJC $(f), 3 d^{10} 4 s^{2}{ }^{1} S$ state; $\mathrm{W}$ : state averaged MR-MP2/SBKJC+ $(d, f)^{6-8}$ for the states $5 d^{4} 6 s^{2}{ }^{5} D, 5 d^{5} 6 s^{1}{ }^{7} S$, and $5 d^{6}$ $\left.{ }^{5} D\right)$.

\begin{tabular}{|l|c|l|c|}
\hline atom & $l$ & \multicolumn{1}{|c|}{$\alpha_{l}$} & method \\
\hline \hline $\mathrm{O}$ & $s, p$ & 0.0845 & $\mathrm{a}$ \\
$\mathrm{O}$ & $d$ & 0.8 & $\mathrm{~b}$ \\
\hline $\mathrm{P}$ & $s, p$ & 0.0348 & $\mathrm{a}$ \\
$\mathrm{P}$ & $d$ & 0.55 & $\mathrm{~b}$ \\
\hline $\mathrm{Zn}$ & $d$ & 0.1088 & $\mathrm{a}$ \\
$\mathrm{Zn}$ & $f$ & 3.117 & $\mathrm{c}$ \\
\hline $\mathrm{W}$ & $s, p$ & 0.0125 & $\mathrm{a}$ \\
$\mathrm{W}$ & $d$ & 0.0175167 & $\mathrm{a}$ \\
$\mathrm{W}$ & $f$ & 0.4456 & $\mathrm{c}$ \\
\hline
\end{tabular}

Table 2: Electronic energies $E_{\text {el }}$ of equilibrium structures.

\begin{tabular}{|l|l|r|}
\hline \multicolumn{1}{|c|}{ compound } & \multicolumn{1}{c|}{ method } & \multicolumn{1}{c|}{$E_{\text {el }} /$ a.u. } \\
\hline$\left[\left(\mathrm{PO}_{4}\right) \mathrm{W}_{12} \mathrm{O}_{36}\right]^{3-}$ & B3LYP/SBKJC $+(d, f)$ & -1462.373061 \\
{$\left[\left(\mathrm{ZnO}_{4}\right) \mathrm{W}_{12} \mathrm{O}_{36}\right]^{3-}$} & B3LYP/SBKJC $+(d, f)$ & -1681.126573 \\
$\mathrm{C}_{33} \mathrm{H}_{48} \mathrm{~N}^{+}$ & B3LYP/SBKJC $(d)$ & -230.650691 \\
$\mathrm{C}_{74} \mathrm{H}_{96} \mathrm{~N}^{+}$ & B3LYP/SBKJC $(d)$ & -485.496912 \\
$\mathrm{C}_{52} \mathrm{H}_{60} \mathrm{NO}_{12}^{+}$ & B3LYP/SBKJC $(d)$ & -531.084066 \\
$\mathrm{C}_{116} \mathrm{H}_{124} \mathrm{NO}_{28}^{+}$ & B3LYP/SBKJC $(d)$ & -1186.522752 \\
\hline
\end{tabular}




\section{A.2 Cartesian coordinates (in $\AA$ ), atom types, and RESP charges for Keggin ions and dendrimers}

\begin{tabular}{|c|c|c|c|c|c|}
\hline Z & $\mathrm{x}$ & $\mathrm{y}$ & $z$ & $\begin{array}{l}\text { atom } \\
\text { type }\end{array}$ & $q / e$ \\
\hline 15 & 0.00000 & 0.00000 & 0.00000 & p5 & 0.015528 \\
\hline 8 & -0.89601 & 0.89601 & -0.89601 & 0 & 0.010652 \\
\hline 8 & 0.89601 & -0.89601 & -0.89601 & o & 0.010652 \\
\hline 8 & 0.89601 & 0.89601 & 0.89601 & o & 0.010652 \\
\hline 8 & -0.89601 & -0.89601 & 0.89601 & o & 0.010652 \\
\hline 74 & -2.54413 & 2.54413 & -0.09476 & $\mathrm{w}$ & 0.004291 \\
\hline 74 & 2.54413 & -2.54413 & -0.09476 & w & 0.004291 \\
\hline 74 & 2.54413 & 2.54413 & 0.09476 & w & 0.004291 \\
\hline 74 & -0.09476 & -2.54413 & 2.54413 & W & 0.004291 \\
\hline 74 & 0.09476 & -2.54413 & -2.54413 & w & 0.004291 \\
\hline 74 & 0.09476 & 2.54413 & 2.54413 & w & 0.00 \\
\hline 74 & -0.09476 & 2.54413 & -2.54413 & w & 0.004291 \\
\hline 74 & -2.54413 & -0.09476 & 2.54413 & w & 0.004291 \\
\hline 74 & 2.54413 & 0.09476 & 2.54413 & W & 0.004291 \\
\hline 74 & -2.54413 & 0.09476 & -2.54413 & w & 0.004291 \\
\hline 74 & 2.54413 & -0.09476 & -2.54413 & $\mathrm{w}$ & 0.004291 \\
\hline 74 & -2.54413 & -2.54413 & 0.09476 & w & 0.004291 \\
\hline 8 & -1.15985 & 1.15985 & 2.99003 & 01 & 0.057050 \\
\hline 8 & 1.15985 & -1.15985 & 2.99003 & 01 & 0.057050 \\
\hline 8 & 1.15985 & 1.15985 & -2.99003 & 01 & 0.057050 \\
\hline 8 & 2.99003 & -1.15985 & 1.15985 & 01 & 0.057050 \\
\hline 8 & -2.99003 & -1.15985 & -1.15985 & 01 & 0.057050 \\
\hline 8 & -2.99003 & 1.15985 & 1.15985 & 01 & 0.057050 \\
\hline 8 & 2.99003 & 1.15985 & -1.15985 & 01 & 0.057050 \\
\hline 8 & -1.15985 & 2.99003 & 1.15985 & 01 & 0.057050 \\
\hline 8 & 1.15985 & -2.99003 & 1.15985 & 01 & 0.057050 \\
\hline 8 & -1.15985 & -2.99003 & -1.15985 & 01 & 0.057050 \\
\hline 8 & 1.15985 & 2.99003 & -1.15985 & 01 & 0.057050 \\
\hline 8 & -1.15985 & -1.15985 & -2.99003 & 01 & 0.057050 \\
\hline 8 & -1.48676 & 1.48676 & -3.35721 & ०2 & -0.034397 \\
\hline 8 & 1.48676 & -1.48676 & -3.35721 & ०2 & -0.034397 \\
\hline 8 & 1.48676 & 1.48676 & 3.35721 & o2 & -0.034397 \\
\hline 8 & -3.35721 & -1.48676 & 1.48676 & o2 & -0.034397 \\
\hline 8 & 3.35721 & -1.48676 & -1.48676 & ०2 & -0.034397 \\
\hline 8 & 3.35721 & 1.48676 & 1.48676 & ०2 & -0.034397 \\
\hline 8 & -3.35721 & 1.48676 & -1.48676 & ०2 & -0.034397 \\
\hline 8 & -1.48676 & -3.35721 & 1.48676 & ०2 & -0.034397 \\
\hline 8 & 1.48676 & 3.35721 & 1.48676 & o2 & -0.034397 \\
\hline
\end{tabular}




$\begin{array}{rrrrrr}8 & -1.48676 & 3.35721 & -1.48676 & \text { o2 } & -0.034397 \\ 8 & 1.48676 & -3.35721 & -1.48676 & \text { o2 } & -0.034397 \\ 8 & -1.48676 & -1.48676 & 3.35721 & \text { o2 } & -0.034397 \\ 8 & -3.73687 & 3.73687 & 0.19524 & \text { ot } & -0.281790 \\ 8 & 3.73687 & -3.73687 & 0.19524 & \text { ot } & -0.281790 \\ 8 & 3.73687 & 3.73687 & -0.19524 & \text { ot } & -0.281790 \\ 8 & 0.19524 & -3.73687 & 3.73687 & \text { ot } & -0.281790 \\ 8 & -0.19524 & -3.73687 & -3.73687 & \text { ot } & -0.281790 \\ 8 & -0.19524 & 3.73687 & 3.73687 & \text { ot } & -0.281790 \\ 8 & 0.19524 & 3.73687 & -3.73687 & \text { ot } & -0.281790 \\ 8 & -3.73687 & 0.19524 & 3.73687 & \text { ot } & -0.281790 \\ 8 & 3.73687 & -0.19524 & 3.73687 & \text { ot } & -0.281790 \\ 8 & -3.73687 & -0.19524 & -3.73687 & \text { ot } & -0.281790 \\ 8 & 3.73687 & 0.19524 & -3.73687 & \text { ot } & -0.281790 \\ 8 & -3.73687 & -3.73687 & -0.19524 & \text { ot } & -0.281790\end{array}$




\begin{tabular}{|c|c|c|c|c|c|}
\hline & $\mathrm{x}$ & $\mathrm{y}$ & $z$ & $\begin{array}{l}\text { atom } \\
\text { type }\end{array}$ & $q / e$ \\
\hline 0 & 0.000000 & .000000 & 0.000000 & $\mathrm{zn}$ & $-1.12<{ }^{2}$ \\
\hline 4 & 0.161054 & -2.497812 & -2.497812 & o & -0.07 \\
\hline 4 & -0.161054 & 2.497812 & -2.497812 & o & -0.074764 \\
\hline 4 & -0.161054 & -2.497812 & 2.497 & o & $-0.0^{\circ}$ \\
\hline 4 & 0.161054 & 2.497812 & 2.497 & 0 & -0.074764 \\
\hline 4 & 2.497812 & 0.1610 & 2.49 & $\mathrm{~W}$ & 1.46 \\
\hline 4 & 2.497812 & -0.161054 & -2.497812 & w & 1.465892 \\
\hline 4 & -2.497812 & $-0.161 c$ & 2.49 & w & 1.46 \\
\hline 4 & -2.497812 & 0.161 & -2.49 & w & 1.4 \\
\hline 4 & -2.497812 & $2.497 \varepsilon$ & -0.16 & $\mathrm{w}$ & 1.46 \\
\hline 74 & 2.497812 & 2.497 & 0.16 & $\mathrm{~W}$ & 1.46 \\
\hline 74 & -2.497812 & -2.497 & 0.1 & $\mathrm{w}$ & 1.46 \\
\hline 74 & & -2.4 & & w & \\
\hline 8 & 1.0 & -1.0 & -1 & w & 1.4 \\
\hline 8 & -1.0 & 1.098 & $-1 . c$ & $\mathrm{w}$ & 1.4 \\
\hline 8 & -1.0 & -1.08 & 1. & W & 1.4 \\
\hline 8 & 1.0 & 1.0 & 1. & $\mathrm{w}$ & 1.4 \\
\hline 8 & -0.2 & -3.704 & -3.7 & 01 & 190 \\
\hline 8 & 0.2 & 3.7 & -3.7 & 01 & -0 \\
\hline 8 & 0.2 & -3.704 & & 01 & -0 \\
\hline 8 & -0.2 & 3.7 & 3.7 & 01 & -0 \\
\hline 8 & 3.7 & -0.203 & & 01 & -0. \\
\hline 8 & 54 & 0.2 & -3.7 & 01 & -0.6 \\
\hline 8 & -3.704154 & 0.2 & & o1 & -0.6 \\
\hline 8 & -3.70 & -0.2 & -3.7 & 01 & -0. \\
\hline 8 & -3.704154 & 3.7 & & 01 & -0 \\
\hline 8 & 3.7 & 3.70 & -0.2 & 01 & -0 \\
\hline 8 & -3.7 & -3.7 & -0 & o1 & -0. \\
\hline 8 & & -3.704 & & 01 & -0. \\
\hline 8 & 1.5 & -1.5 & -3. & o2 & -0.4 \\
\hline 8 & -1.547195 & 1.5471 & -3.49 & o2 & -0.4 \\
\hline 8 & -1 & & & o2 & -0 \\
\hline 8 & 1.547195 & 1.547 & 3.4 & ०2 & -0.484136 \\
\hline 8 & 3.4 & 1.5 & & o2 & -0.4 \\
\hline 8 & 3.497044 & -1.547195 & -1.547195 & o2 & -0.484136 \\
\hline 8 & & -1.5471 & & o2 & -0.484136 \\
\hline 8 & -3.497044 & 1.547195 & -1.547195 & o2 & -0.484136 \\
\hline 8 & -1.54719 & 3.497044 & -1.547195 & o2 & -0.484136 \\
\hline 8 & 1.547195 & 3.497044 & 1.547195 & 02 & -0.484136 \\
\hline 8 & -1.547195 & -3.497044 & 1.547195 & o2 & -0.484136 \\
\hline 8 & 1.547195 & -3.497044 & -1.547195 & o2 & -0.484136 \\
\hline
\end{tabular}




\begin{tabular}{|c|c|c|c|c|c|}
\hline & -1.101466 & -1.101466 & -2.937338 & ot & -0.683849 \\
\hline & 1.101466 & 1.101466 & -2.937338 & ot & -0.683849 \\
\hline & 1.101466 & -1.101466 & 2.937338 & ot & 3849 \\
\hline & -1.101466 & 1.101466 & 2.937338 & ot & 3849 \\
\hline & 2.937338 & -1.101466 & 1.101466 & ot & 849 \\
\hline & 2.937338 & 1.101466 & -1.101466 & ot & -0.683849 \\
\hline & -2.937338 & 1.101466 & 1.101466 & ot & -0.683849 \\
\hline & -2.937338 & -1.101466 & -1.101466 & ot & -0.683849 \\
\hline & -1.101466 & 2.937338 & 1.101466 & ot & -0.683849 \\
\hline & 1.101466 & 2.937338 & -1.101466 & ot & -0.683849 \\
\hline & -1.101466 & -2.937338 & -1.101466 & ot & -0.683849 \\
\hline & 1.101466 & -2.937338 & 1.101466 & ot & -0.683849 \\
\hline
\end{tabular}




\begin{tabular}{|c|c|c|c|c|c|}
\hline \multicolumn{6}{|c|}{$\mathrm{C}_{33} \mathbf{H}_{48} \mathbf{N}^{+}$} \\
\hline & $\mathrm{x}$ & y & $z$ & $\begin{array}{l}\text { atom } \\
\text { type }\end{array}$ & $q / e$ \\
\hline & -1.158658 & -0.617081 & 1.243620 & $\mathrm{ca}$ & 0.005383 \\
\hline & -2.411947 & -1.277632 & 1.190750 & $\mathrm{ca}$ & -0.105079 \\
\hline & -0.550423 & -0.287397 & 0.000000 & $\mathrm{ca}$ & -0.005614 \\
\hline & -0.536133 & -0.280546 & 2.557243 & $\mathrm{ca}$ & 0.118605 \\
\hline & -3.001562 & -1.581729 & 0.000000 & na & 0.235146 \\
\hline & -1.158658 & -0.617081 & -1.243620 & $\mathrm{ca}$ & 0.005383 \\
\hline & 0.869857 & -0.373712 & 2.713793 & $\mathrm{ca}$ & -0.283779 \\
\hline & -1.352802 & 0.134731 & 3.650343 & $\mathrm{ca}$ & -0.283779 \\
\hline & -2.946576 & -1.586595 & 2.088857 & h4 & 0.164526 \\
\hline & 0.388525 & 0.268526 & 0.000000 & ha & 0.125428 \\
\hline & -2.411947 & -1.277632 & -1.190750 & $\mathrm{ca}$ & -0.10 \\
\hline & -0.536133 & -0.280546 & -2.557243 & $\mathrm{ca}$ & 0.118605 \\
\hline & -4.339994 & -2.247877 & 0.000000 & c3 & -0.491629 \\
\hline & 1.487322 & -0.060872 & 3.956072 & $\mathrm{ca}$ & 0.0 \\
\hline & -0.772954 & 0.461280 & 4.899140 & $\mathrm{ca}$ & 0.055070 \\
\hline & 1.477886 & -0.723468 & 1.876669 & ha & 0.142592 \\
\hline & -2.427643 & 0.243450 & 3501 & ha & 0.142592 \\
\hline & 0.869857 & -0.373 & & $\mathrm{ca}$ & -0.2 \\
\hline & -1.352802 & 0.134731 & -3.650 & $\mathrm{ca}$ & -0.283779 \\
\hline & 0.644576 & 0.3506 & 5.020 & $\mathrm{ca}$ & -0.277093 \\
\hline & -1.610954 & 0.936832 & 84 & c3 & 0.630823 \\
\hline & 3.023782 & -0.190248 & 4.104477 & c3 & 0.630823 \\
\hline & -2.946576 & -1.586595 & -2.088857 & h4 & 0.164526 \\
\hline & -5.127714 & -1.4793 & & h1 & 0.208137 \\
\hline 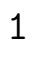 & -4.427529 & -2.875913 & -0.895 & h1 & 0.208137 \\
\hline & -4.427529 & -2.875913 & 0.895 & h1 & 0.20 \\
\hline & 1.487322 & -0.060872 & -3.956072 & $\mathrm{ca}$ & 0.055070 \\
\hline & -0.772954 & 0.461280 & -4.899140 & $\mathrm{ca}$ & 0.055070 \\
\hline & -3.129587 & 0.989235 & 5.796575 & c3 & -0.612975 \\
\hline & 3.519398 & 0.207779 & 5519 & c3 & -0.612975 \\
\hline & 1.477886 & -0.723468 & -1.876669 & ha & 0.142592 \\
\hline & -2.427643 & 0.243450 & -3.501088 & ha & 0.142592 \\
\hline , & 1.099142 & 0.597514 & 5.978928 & ha & 0.157506 \\
\hline & -1.149767 & 2.366503 & 6.525619 & c3 & -0.612975 \\
\hline 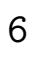 & 3.720484 & 0.739369 & 3.066070 & c3 & -0.612975 \\
\hline 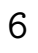 & -1.394446 & -0.044793 & 7.301924 & c3 & -0.612975 \\
\hline & 3.440379 & -1.668446 & 3.839757 & c3 & -0.612975 \\
\hline 6 & 0.644576 & 0.350693 & -5.020410 & $\mathrm{ca}$ & -0.277093 \\
\hline 5 & -1.610954 & 0.936832 & -6.110984 & c3 & 0.630823 \\
\hline 6 & 3.023782 & -0.190248 & -4.104477 & c3 & 0.630823 \\
\hline 4 & -3.678800 & 1.327331 & 6.690393 & hc & 0.154485 \\
\hline
\end{tabular}




$\begin{array}{rrrrrr}1 & 4.615781 & 0.102152 & 5.566863 & \text { hc } & 0.154485 \\ 1 & -1.301800 & 3.087872 & 5.703984 & \text { hc } & 0.154485 \\ 1 & -0.083178 & 2.391666 & 6.802940 & \text { hc } & 0.154485 \\ 1 & -1.730181 & 2.713743 & 7.397903 & \text { hc } & 0.154485 \\ 1 & -3.528956 & -0.003275 & 5.522692 & \text { hc } & 0.154485 \\ 1 & -3.359922 & 1.698782 & 4.982923 & \text { hc } & 0.154485 \\ 1 & 3.450482 & 1.796325 & 3.233701 & \text { hc } & 0.154485 \\ 1 & 3.445410 & 0.480954 & 2.029234 & \text { hc } & 0.154485 \\ 1 & 4.817172 & 0.649154 & 3.151301 & \text { hc } & 0.154485 \\ 1 & 3.093007 & -0.439974 & 6.304746 & \text { hc } & 0.154485 \\ 1 & 3.278781 & 1.256926 & 5.762622 & \text { hc } & 0.154485 \\ 1 & -0.336294 & -0.100205 & 7.605543 & \text { hc } & 0.154485 \\ 1 & -1.724465 & -1.065888 & 7.042287 & \text { hc } & 0.154485 \\ 1 & -1.975647 & 0.290072 & 8.178494 & \text { hc } & 0.154485 \\ 1 & 3.152660 & -2.005563 & 2.829443 & \text { hc } & 0.154485 \\ 1 & 2.970330 & -2.350512 & 4.569103 & \text { hc } & 0.154485 \\ 1 & 4.535363 & -1.775818 & 3.928281 & \text { hc } & 0.154485 \\ 6 & -3.129587 & 0.989235 & -5.796575 & \mathrm{c} 3 & -0.612975 \\ 6 & 3.519398 & 0.207779 & -5.519963 & \mathrm{c} 3 & -0.612975 \\ 1 & 1.099142 & 0.597514 & -5.978928 & \mathrm{hc} & 0.157506 \\ 6 & -1.394446 & -0.044793 & -7.301924 & \mathrm{c} 3 & -0.612975 \\ 6 & 3.440379 & -1.668446 & -3.839757 & \mathrm{c} 3 & -0.612975 \\ 6 & -1.149767 & 2.366503 & -6.525619 & \mathrm{c} 3 & -0.612975 \\ 6 & 3.720484 & 0.739369 & -3.066070 & \mathrm{c} 3 & -0.612975 \\ 1 & -3.678800 & 1.327331 & -6.690393 & \mathrm{hc} & 0.154485 \\ 1 & 4.615781 & 0.102152 & -5.566863 & \mathrm{hc} & 0.154485 \\ 1 & -1.724465 & -1.065888 & -7.042287 & \mathrm{hc} & 0.154485 \\ 1 & -0.336294 & -0.100205 & -7.605543 & \mathrm{hc} & 0.154485 \\ 1 & -1.975647 & 0.290072 & -8.178494 & \mathrm{hc} & 0.154485 \\ 1 & -3.359922 & 1.698782 & -4.982923 & \mathrm{hc} & 0.154485 \\ 1 & -3.528956 & -0.003275 & -5.522692 & \mathrm{hc} & 0.154485 \\ 1 & 2.970330 & -2.350512 & -4.569103 & \mathrm{hc} & 0.154485 \\ 1 & 3.152660 & -2.005563 & -2.829443 & \mathrm{hc} & 0.154485 \\ 1 & 4.535363 & -1.775818 & -3.928281 & \mathrm{hc} & 0.154485 \\ 1 & 3.278781 & 1.256926 & -5.762622 & \mathrm{hc} & 0.154485 \\ 1 & 3.093007 & -0.439974 & -6.304746 & \mathrm{hc} & 0.154485 \\ 1 & -0.083178 & 2.391666 & -6.802940 & \mathrm{hc} & 0.154485 \\ 1 & -1.301800 & 3.087872 & -5.703984 & \mathrm{hc} & 0.154485 \\ 1 & -1.730181 & 2.713743 & -7.397903 & \mathrm{hc} & 0.154485 \\ 1 & 3.445410 & 0.480954 & -2.029234 & \mathrm{hc} & 0.154485 \\ 1 & 3.450482 & 1.796325 & -3.233701 & \mathrm{hc} & 0.154485 \\ 1 & 4.817172 & 0.649154 & -3.151301 & \mathrm{hc} & 0.154485\end{array}$




\begin{tabular}{|c|c|c|c|c|c|}
\hline \multicolumn{6}{|c|}{$\mathbf{C}_{74} \mathbf{H}_{96} \mathbf{N}^{+}(3 \mathbf{b})$} \\
\hline Z & $\mathrm{x}$ & $\mathrm{y}$ & $z$ & $\begin{array}{l}\text { atom } \\
\text { type }\end{array}$ & $q / e$ \\
\hline 6 & 2.051164 & 1.235791 & 0.000000 & $\mathrm{ca}$ & 0.120402 \\
\hline 6 & 3.461678 & 1.190327 & 0.000000 & $\mathrm{ca}$ & -0.185676 \\
\hline - $>$ & 1.352320 & -0.000147 & 0.000000 & $\mathrm{ca}$ & -0.103658 \\
\hline 6 & 1.349765 & 2.563027 & 0.000000 & $\mathrm{ca}$ & 0.083949 \\
\hline 1 & 4.134338 & 0.002451 & 0.000000 & na & 0.306543 \\
\hline 6 & 2.054372 & -1.237071 & 0.000000 & $\mathrm{ca}$ & 0.126462 \\
\hline 6 & 1.039277 & 3.188741 & 1.233382 & $\mathrm{ca}$ & -0.244500 \\
\hline 6 & 1.039277 & 3.188741 & -1.233382 & $\mathrm{ca}$ & -0.244500 \\
\hline 1 & 0.259568 & -0.001379 & 0.000 & ha & 0.149863 \\
\hline 6 & 3.462229 & -1.188227 & 0.000000 & $\mathrm{ca}$ & -0.185676 \\
\hline 6 & 1.353528 & -2.564405 & 0.000000 & $\mathrm{ca}$ & 0.083949 \\
\hline 6 & 5.629562 & -0.018829 & 0.000000 & c3 & -0.632068 \\
\hline 6 & & 4.460 & 1.246 & $\mathrm{ca}$ & 0.0 \\
\hline 6 & 0.395962 & 4.460 & -1.246 & $\mathrm{ca}$ & 0.0 \\
\hline 1 & 1.312583 & 2.7092 & 2.176 & ha & 0.124473 \\
\hline 1 & 1.312583 & 2.709 & -2.176 & ha & 0.124473 \\
\hline 6 & 1.042116 & -3.189544 & 1.233 & $\mathrm{ca}$ & -0.244500 \\
\hline 6 & 1.042116 & -3.189544 & -1.233 & $\mathrm{ca}$ & -0.244500 \\
\hline 6 & 0.080102 & 5.0702 & 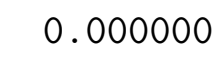 & $\mathrm{ca}$ & -0.130599 \\
\hline 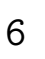 & 0.050810 & 5.1310 & 2.541 & $\mathrm{ca}$ & 0.126699 \\
\hline 6 & 0.050810 & 5.131 & -2.5 & $\mathrm{ca}$ & 0.126699 \\
\hline 1 & 4.073543 & -2.0923 & 0.000 & h4 & 0.1 \\
\hline 1 & 5.983004 & -0.540030 & 0.901 & h1 & 0.251057 \\
\hline 1 & 5.983004 & -0.540030 & -0.901 & h1 & 0.251057 \\
\hline 1 & 6.001856 & 1.012713 & 0 & h1 & 0.251057 \\
\hline 6 & 0.397243 & -4.460478 & & $\mathrm{ca}$ & 0.060950 \\
\hline 6 & 0.397243 & -4.460478 & -1.246 & $\mathrm{ca}$ & 0.060950 \\
\hline 6 & -0.434052 & 4.3708 & 3.639 & $\mathrm{ca}$ & -0.282333 \\
\hline 6 & -0.434052 & 4.370814 & -3.639 & $\mathrm{ca}$ & -0.28233 \\
\hline 6 & 0.206577 & 6.535985 & 2.683 & $\mathrm{ca}$ & -0.282333 \\
\hline 6 & 0.206577 & 6.535985 & -2.683234 & $\mathrm{ca}$ & -0.28233 \\
\hline 1 & 1.315890 & -2.7102 & & ha & 0.1 \\
\hline 1 & 1.315890 & -2.710296 & -2.176233 & ha & 0.12447 \\
\hline 1 & -0.462410 & 6.017431 & 0.000000 & ha & 0.112708 \\
\hline 6 & 0.080629 & -5.069902 & 0.000000 & $\mathrm{ca}$ & -0.130599 \\
\hline 6 & 0.051579 & -5.130797 & 2.541633 & $\mathrm{ca}$ & 0.126699 \\
\hline 6 & 0.051579 & -5.130797 & -2.541633 & $\mathrm{ca}$ & 0.12669 \\
\hline 6 & -0.766434 & 4.989352 & 4.872976 & $\mathrm{ca}$ & 0.03819 \\
\hline 6 & -0.766434 & 4.989352 & -4.872976 & $\mathrm{ca}$ & 0.038190 \\
\hline 6 & -0.111997 & 7.192868 & 3.900229 & $\mathrm{ca}$ & 0.038190 \\
\hline 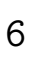 & -0.111997 & 7.192868 & -3.900229 & $\mathrm{ca}$ & 0.03819 \\
\hline
\end{tabular}




\begin{tabular}{|c|c|c|c|c|c|}
\hline & -0.583154 & 3.296464 & 3.509432 & ha & 0.143083 \\
\hline & -0.583154 & 3.296464 & -3.509432 & ha & 0.143083 \\
\hline & 0.604876 & 7.106940 & 1.841810 & 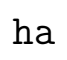 & 0.143083 \\
\hline & 0.604876 & 7.106940 & -1.841810 & 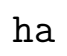 & 0.143083 \\
\hline & 0.205709 & -6.535891 & 2.683114 & & -0.28233 \\
\hline & 0.205709 & -6.535891 & -2.683114 & & -0.28233 \\
\hline & -0.432169 & -4.370026 & 3.639872 & & -0.28233 \\
\hline & -0.432169 & -4.370026 & -3.639872 & & -0.28233 \\
\hline & -0.595148 & 6.397947 & 4.976835 & & -0.29147 \\
\hline & -0.595148 & 6.397947 & -4.976835 & & -0.29147 \\
\hline & 0.083204 & 8.725684 & 4.018981 & 3 & 0.67442 \\
\hline & 0.083204 & 8.725684 & -4.018981 & & 0.67442 \\
\hline & -1.314416 & 4.129591 & 6.040396 & & 0.67442 \\
\hline & -1.314416 & 4.129591 & -6.040396 & 3 & 0.67442 \\
\hline & -0.463006 & -6.016415 & 0.000000 & & 0.11270 \\
\hline & -0.113443 & -7.192488 & 3.900115 & & 0.0381 \\
\hline & -0.113443 & -7.192488 & -3.900115 & & 0.03819 \\
\hline & -0.765077 & -4.988260 & 4.873158 & & 0.0381 \\
\hline & -0.765077 & -4.988260 & -4.873158 & & 0.0381 \\
\hline & -0.274896 & 3.027995 & 6.405252 & 3 & -0.633989 \\
\hline & -0.274896 & 3.02 & -6.405252 & 3 & -0.6335 \\
\hline & -1 & 4.9 & 7.317408 & & -0 . \\
\hline & -1.596244 & 4.96 & -7.317408 & 3 & -0.633989 \\
\hline & -0.329043 & 9.274366 & 5.410893 & & -0.633989 \\
\hline & -0.329043 & 9.274366 & -5.410893 & 3 & -0.633989 \\
\hline & 1.584445 & 9.072939 & 3.786849 & & -0.633989 \\
\hline & 1.584445 & 9.0 & -3.786849 & 3 & -0.633989 \\
\hline & -2.647759 & 3.4 & 5.598975 & 3 & -0.633989 \\
\hline & -2.6 & 3.4 & -5.598975 & & -0.633 \\
\hline & -0.784803 & 9.44 & 2.941022 & 3 & -0.633989 \\
\hline & -0.784803 & 9.44 & -2.941022 & & -0.633989 \\
\hline & -0.845239 & 6.8 & 5.915249 & & 0.132315 \\
\hline & -0.845239 & 6.886779 & -5.915249 & & 0.13231 \\
\hline & -0.595451 & -6.397068 & 4.976868 & $a$ & -0.291474 \\
\hline & -0.595451 & -6.3 & -4.976868 & & -0.29147 \\
\hline & 0.079961 & -8.72 & 4.018708 & 3 & 0.674421 \\
\hline & 0.079961 & -8.725548 & -4.018708 & & 0.67442 \\
\hline & -1.311760 & -4.127923 & 6.040765 & & 0.6744 \\
\hline & -1.311760 & -4.127923 & -6.040765 & c3 & 0.67442 \\
\hline & 0.679434 & 3.476848 & 6.731823 & & 0.15472 \\
\hline & 0.679434 & 3.476848 & -6.731823 & & 0.15472 \\
\hline & -0.060323 & 2.365943 & 5.549235 & & 0.15472 \\
\hline & -0.060323 & 2.365943 & -5.549235 & & 0.15472 \\
\hline & -0.656671 & 2.398397 & 7.228573 & c & 0.15472 \\
\hline & -0.656671 & 2.398397 & -7.228573 & & 0.1547 \\
\hline
\end{tabular}




\begin{tabular}{|c|c|c|c|c|c|}
\hline & -2.360890 & 5.740779 & 7.142131 & hc & 0.154725 \\
\hline & -2.360890 & 5.740779 & -7.142131 & hc & 0.154725 \\
\hline & -0.684595 & 5.456025 & 7.698948 & hc & 0.15472 \\
\hline & -0.684595 & 5.456025 & -7.698948 & & 0.15472 \\
\hline & -1.974804 & 4.303448 & 8.114539 & & 0.15472 \\
\hline & -1.974804 & 4.303448 & -8.114539 & & 0.1547 \\
\hline & 0.275612 & 8.836297 & 6.223277 & & 0.15472 \\
\hline & 0.275612 & 8.836297 & -6.223277 & c & 0.15472 \\
\hline & -1.394071 & 9.086351 & 5.629508 & & 0.1547 \\
\hline & -1.394071 & 9.086351 & -5.629508 & & 0.15472 \\
\hline & -0.174311 & 10.366093 & 5.436306 & & 0.15472 \\
\hline & -0.174311 & 10.366093 & -5.436306 & & 0.15472 \\
\hline & 1.934388 & 8.744283 & 2.793921 & & 0.15472 \\
\hline & 1.934388 & 8.744283 & -2.793921 & & 0.15472 \\
\hline & 2.224153 & 8.589575 & 4.545760 & & 0.15472 \\
\hline & 2.224153 & 8.589575 & -4.545760 & & 0.15472 \\
\hline & 1.739328 & 10.164462 & 3.853451 & & 0 . \\
\hline & 1.739328 & 10.164462 & -3.853451 & & 0.15472 \\
\hline & -2.509912 & 2.811229 & 790 & & 0.15472 \\
\hline & -2.509912 & 2.811229 & -4.713790 & & 0.15472 \\
\hline & -3.409144 & 4.212522 & 5.346074 & & 0.15472 \\
\hline & -3.409144 & 4.212522 & -5.346074 & & 0.154725 \\
\hline & -3.047777 & 2.826015 & 6.414523 & & 0.15472 \\
\hline & -3 & 2.82 & -6.414523 & & \\
\hline & -1.856757 & 9.221277 & 3.085661 & & 0.15472 \\
\hline & -1.856757 & 9.221277 & -3.085661 & & 0.15472 \\
\hline & -0.512872 & 9.128295 & 1.919031 & & 0.154725 \\
\hline & -0.512872 & 9.128295 & -1.919031 & & 0.15472 \\
\hline & -0.647932 & 10.535368 & 3.004066 & & 0.154725 \\
\hline & -0.647932 & 10.535368 & -3.004066 & & 0.15472 \\
\hline & 1.580720 & -9.0 & 3.78 & & -0.633989 \\
\hline & 1.580720 & -9.074589 & -3.786134 & & -0.633989 \\
\hline & -0.332559 & -9.273839 & 5.410697 & & $-0.63398 s$ \\
\hline & -0.332559 & -9.273839 & -5.410697 & & -0.633989 \\
\hline & -0.789207 & -9.440051 & 2.940924 & & -0.63398 \\
\hline & -0.789207 & -9.440051 & -2.940924 & 3 & -0.633989 \\
\hline & -1.594838 & -4.963467 & 7.317604 & & -0.63398 \\
\hline & -1.594838 & -4.963467 & -7.317604 & 3 & -0.633989 \\
\hline & -0.270556 & -3.027977 & 6.405799 & c3 & -0.633989 \\
\hline & -0.270556 & -3.027977 & -6.405799 & & -0.63398 \\
\hline & -2.644095 & -3.450852 & 5.599527 & . & -0.633989 \\
\hline & -2.644095 & -3.450852 & -5.599527 & & -0.633989 \\
\hline & 2.221213 & -8.592075 & 4.544924 & & 0.154725 \\
\hline & 2.221213 & -8.592075 & -4.544924 & & 0.15472 \\
\hline & 1.930799 & -8.746268 & 2.793143 & & 0.15472 \\
\hline
\end{tabular}




$\begin{array}{rrrrll}1 & 1.930799 & -8.746268 & -2.793143 & \text { hc } & 0.154725 \\ 1 & 1.734302 & -10.166302 & 3.852593 & \text { hc } & 0.154725 \\ 1 & 1.734302 & -10.166302 & -3.852593 & \text { hc } & 0.154725 \\ 1 & -1.397330 & -9.084684 & 5.629576 & \text { hc } & 0.154725 \\ 1 & -1.397330 & -9.084684 & -5.629576 & \text { hc } & 0.154725 \\ 1 & 0.272764 & -8.836461 & 6.222960 & \text { hc } & 0.154725 \\ 1 & 0.272764 & -8.836461 & -6.222960 & \text { hc } & 0.154725 \\ 1 & -0.179008 & -10.365733 & 5.436028 & \text { hc } & 0.154725 \\ 1 & -0.179008 & -10.365733 & -5.436028 & \text { hc } & 0.154725 \\ 1 & -0.517204 & -9.127223 & 1.918887 & \text { hc } & 0.154725 \\ 1 & -0.517204 & -9.127223 & -1.918887 & \text { hc } & 0.154725 \\ 1 & -1.860861 & -9.218762 & 3.085900 & \text { hc } & 0.154725 \\ 1 & -1.860861 & -9.218762 & -3.085900 & \text { hc } & 0.154725 \\ 1 & -0.653597 & -10.534255 & 3.003806 & \text { hc } & 0.154725 \\ 1 & -0.653597 & -10.534255 & -3.003806 & \text { hc } & 0.154725 \\ 1 & -0.683924 & -5.455606 & 7.699103 & \text { hc } & 0.154725 \\ 1 & -0.683924 & -5.455606 & -7.699103 & \text { hc } & 0.154725 \\ 1 & -2.360585 & -5.737836 & 7.142117 & \text { hc } & 0.154725 \\ 1 & -2.360585 & -5.737836 & -7.142117 & \text { hc } & 0.154725 \\ 1 & -1.972482 & -4.301274 & 8.114863 & \text { hc } & 0.154725 \\ 1 & -1.972482 & -4.301274 & -8.114863 & \text { hc } & 0.154725 \\ 1 & -0.055002 & -2.366085 & 5.549902 & \text { hc } & 0.154725 \\ 1 & -0.055002 & -2.366085 & -5.549902 & \text { hc } & 0.154725 \\ 1 & 0.683094 & -3.478360 & 6.732253 & \text { hc } & 0.154725 \\ 1 & 0.683094 & -3.478360 & -6.732253 & \text { hc } & 0.154725 \\ 1 & -0.651329 & -2.397955 & 7.229258 & \text { hc } & 0.154725 \\ 1 & -0.651329 & -2.397955 & -7.229258 & \text { hc } & 0.154725 \\ 1 & -3.406624 & -4.207511 & 5.346417 & \text { hc } & 0.154725 \\ 1 & -3.406624 & -4.207511 & -5.346417 & \text { hc } & 0.154725 \\ 1 & -2.505299 & -2.807373 & 4.714535 & \text { hc } & 0.154725 \\ 1 & -2.505299 & -2.807373 & -4.714535 & \text { hc } & 0.154725 \\ 1 & -3.043154 & -2.821863 & 6.415261 & \text { hc } & 0.154725 \\ 1 & -3.043154 & -2.821863 & -6.415261 & \text { hc } & 0.154725 \\ 1 & 0.603171 & -7.107254 & 1.841572 & \text { ha } & 0.143083 \\ 1 & 0.603171 & -7.107254 & -1.841572 & \text { ha } & 0.143083 \\ 1 & -0.580017 & -3.295490 & 3.509733 & \text { ha } & 0.143083 \\ 1 & -0.580017 & -3.295490 & -3.509733 & \text { ha } & 0.143083 \\ 1 & -0.845939 & -6.885667 & 5.915299 & \text { ha } & 0.132315 \\ 1 & -0.845939 & -6.885667 & -5.915299 & \text { ha } & 0.132315 \\ 1 & 4.066527 & 2.098059 & 0.000000 & \text { h4 } & 0.183634\end{array}$




\begin{tabular}{|c|c|c|c|c|c|}
\hline \multicolumn{6}{|c|}{$\mathbf{C}_{52} \mathbf{H}_{60} \mathrm{NO}_{12}^{+}(\mathbf{4 a})$} \\
\hline Z & $\mathrm{x}$ & $\mathrm{y}$ & z & $\begin{array}{l}\text { atom } \\
\text { type }\end{array}$ & $\mathrm{q} / \mathrm{e}$ \\
\hline 7 & 0.000000 & 0.000000 & 1.357843 & $\mathrm{n} 4$ & 0.394712 \\
\hline 6 & 1.232109 & 0.000000 & 0.479574 & c3 & -0.323488 \\
\hline 6 & -1.232109 & 0.000000 & 0.479574 & c3 & -0.323488 \\
\hline 6 & 0.000000 & 1.246233 & 2.295954 & c3 & -0.433898 \\
\hline 6 & 0.000000 & -1.246233 & 2.295954 & c3 & -0.433898 \\
\hline 6 & 0.000000 & 2.598757 & 1.603726 & $\mathrm{ca}$ & 0.468873 \\
\hline 6 & 0.000000 & -2.598757 & 1.603726 & $\mathrm{ca}$ & 0.468873 \\
\hline 1 & -0.894073 & 1.125928 & 2.924136 & $\mathrm{hx}$ & 0.178589 \\
\hline 1 & 0.894073 & 1.125928 & 2.924136 & $\mathrm{hx}$ & 0.178589 \\
\hline 1 & 0.894073 & -1.125928 & 2.924136 & $\mathrm{hx}$ & 0.178589 \\
\hline 1 & -0.894073 & -1.125928 & 2.924136 & $\mathrm{hx}$ & 0.178589 \\
\hline 1 & -2.125242 & 0.000000 & 1.120036 & $\mathrm{hx}$ & 0.148425 \\
\hline 1 & -1.2215 & & & $\mathrm{hx}$ & 0.14 \\
\hline 1 & -1.221516 & -0.903124 & -0.142 & $\mathrm{hx}$ & 0.148425 \\
\hline 1 & 2.125242 & 0.000 & 1.120 & $\mathrm{hx}$ & 0.148425 \\
\hline 1 & 1.221516 & -0.903124 & -0.142 & $\mathrm{hx}$ & 0.148425 \\
\hline 1 & 1.221516 & 0.903124 & -0.142165 & $\mathrm{hx}$ & 0.148425 \\
\hline 6 & 1.232857 & 3.236301 & 1.313149 & $\mathrm{ca}$ & -0.564543 \\
\hline 6 & -1.232857 & 3.236301 & 49 & $\mathrm{ca}$ & -0.564543 \\
\hline 6 & 1.232857 & -3.236301 & 1.313 & $\mathrm{ca}$ & -0.564543 \\
\hline 6 & -1.232857 & -3.236301 & 1.313 & $\mathrm{ca}$ & -0.564543 \\
\hline 6 & 1.229708 & 4.521 & 0.701 & $\mathrm{ca}$ & 0.406225 \\
\hline 6 & -1.229708 & 4.521565 & 0.701658 & $\mathrm{ca}$ & 0.406225 \\
\hline 1 & 2.194067 & 2.785018 & 1.569 & ha & 0.199634 \\
\hline 1 & -2.194067 & 2.785018 & 1560 & ha & 0.199634 \\
\hline 6 & 1.229708 & -4.521 & & $\mathrm{ca}$ & 0.406225 \\
\hline 6 & -1.229708 & -4.521 & 0.701 & $\mathrm{ca}$ & 0.406225 \\
\hline 1 & 2.194067 & -2.785 & 1. & ha & 0.199634 \\
\hline 1 & -2.194067 & -2.785 & 1.569 & ha & 0.199634 \\
\hline 6 & 0.000000 & 5.169563 & 0.388788 & $\mathrm{ca}$ & -0.351001 \\
\hline 8 & 2.462894 & 5.052607 & 0.457757 & os & -0.272225 \\
\hline 8 & -2.462894 & & & os & -0.272225 \\
\hline 6 & 0.000000 & -5.169563 & 0.388788 & $\mathrm{ca}$ & -0.351001 \\
\hline 8 & 2.462894 & -5.052607 & 0.457757 & os & -0.272225 \\
\hline 8 & -2.462894 & -5.052607 & 0.457757 & os & -0.272225 \\
\hline 1 & 0.000000 & 6.152548 & -0.074172 & ha & 0.094868 \\
\hline 1 & 0.000000 & -6.152548 & -0.074172 & ha & 0.094868 \\
\hline 6 & 2.540546 & -6.386894 & -0.123451 & c3 & 0.029885 \\
\hline 6 & 2.540546 & 6.386894 & -0.123451 & c3 & 0.029885 \\
\hline 6 & -2.540546 & -6.386894 & -0.123451 & c3 & 0.029885 \\
\hline 6 & -2.540546 & 6.386894 & -0.123451 & c3 & 0.029885 \\
\hline
\end{tabular}




\begin{tabular}{|c|c|c|c|c|c|}
\hline & 4.005171 & -6.758258 & -0.268443 & $\mathrm{ca}$ & 0.175378 \\
\hline & 2.018231 & -7.094926 & 0.544119 & h1 & 0.097371 \\
\hline & 2.038796 & -6.377334 & -1.107337 & h1 & 0.097371 \\
\hline & 4.005171 & 6.758258 & -0.268443 & $\mathrm{ca}$ & 0.175378 \\
\hline & 2.038796 & 6.377334 & -1.107337 & h1 & 0.097371 \\
\hline & 2.018231 & 7.094926 & 0.544119 & h1 & 0.09737 \\
\hline & 4.005171 & -6.758258 & -0.268443 & $\mathrm{ca}$ & .17537 \\
\hline & 2.038796 & -6.377334 & -1.107337 & h1 & 0.097371 \\
\hline & 2.018231 & -7.094926 & 0.544119 & h1 & 0.097371 \\
\hline & -4.005171 & 6.758258 & -0.268443 & $\mathrm{ca}$ & 0.175378 \\
\hline & -2.018231 & 7.094926 & 0.544119 & h1 & 0.097371 \\
\hline & -2.038796 & 6.377334 & -1.107337 & h1 & 0.097371 \\
\hline & 4.681300 & -6.477282 & -1.480844 & $\mathrm{ca}$ & -0.493481 \\
\hline & 4.679473 & -7.375524 & 0.813423 & $\mathrm{ca}$ & -0.493481 \\
\hline & 4.681300 & 6.477282 & -1.480844 & $\mathrm{ca}$ & -0.493481 \\
\hline & 4.679473 & 7.375524 & 0.813423 & $\mathrm{ca}$ & -0.493481 \\
\hline & -4.681300 & -6.477282 & -1.480844 & $\mathrm{ca}$ & -0.493481 \\
\hline & -4.679473 & -7.375524 & 0.813423 & $\mathrm{ca}$ & -0.493481 \\
\hline & -4.681300 & 6.477282 & -1.480844 & $\mathrm{ca}$ & -0 \\
\hline & -4.679473 & 7.375524 & 0.813423 & $\mathrm{ca}$ & -0.493481 \\
\hline & 6.054601 & -6.815675 & -1.606124 & $\mathrm{ca}$ & 0.456160 \\
\hline & 6.052754 & -7.708821 & 0.677713 & $\mathrm{ca}$ & 0.456160 \\
\hline & 4.172053 & -6.008009 & -2.325523 & ha & 0.202162 \\
\hline & 4.169133 & -7.602716 & 1.751959 & ha & 0.202162 \\
\hline & 6.054601 & 6.815675 & -1.606124 & $\mathrm{ca}$ & 0.456160 \\
\hline & 6.052754 & 7.708821 & 0.677713 & $\mathrm{ca}$ & 0. \\
\hline & 4.172053 & 6.008009 & -2.325523 & ha & 0.202162 \\
\hline & 4.169133 & 7.602716 & 1.751959 & ha & 0.20216 \\
\hline & -6.054601 & -6.815675 & -1.606124 & $\mathrm{ca}$ & 0.456160 \\
\hline & -6.052754 & -7.708821 & 0.677713 & $\mathrm{ca}$ & 0.456160 \\
\hline & -4.172053 & -6.008009 & -2.325523 & ha & 0.202162 \\
\hline & -4.169133 & -7.602716 & 1.751959 & ha & 0.202162 \\
\hline & -6.054601 & 6.815675 & -1.606124 & $\mathrm{ca}$ & 0.456160 \\
\hline & -6.052754 & 7.708821 & 0.677713 & $\mathrm{ca}$ & 0.456160 \\
\hline . & -4.172053 & 6.008009 & -2.325523 & ha & 0.202162 \\
\hline & -4.169133 & 7.602716 & 1.751959 & ha & 0.202162 \\
\hline & 6.753650 & -7.434990 & -0.531510 & $\mathrm{ca}$ & -0.400318 \\
\hline & 6.630003 & -6.507494 & -2.815811 & os & -0.324624 \\
\hline & 6.626541 & -8.303330 & 1.775479 & os & -0.324624 \\
\hline & 6.753650 & 7.434990 & -0.531510 & $\mathrm{ca}$ & -0.400318 \\
\hline & 6.630003 & 6.507494 & -2.815811 & os & -0.324624 \\
\hline & 6.626541 & 8.303330 & 1.775479 & os & -0.324624 \\
\hline 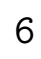 & -6.753650 & -7.434990 & -0.531510 & $\mathrm{ca}$ & -0.400318 \\
\hline & -6.630003 & -6.507494 & -2.815811 & os & -0.324624 \\
\hline & -6.626541 & -8.303330 & 1.775479 & os & -0.32462 \\
\hline
\end{tabular}




$\begin{array}{rrrrrr}6 & -6.753650 & 7.434990 & -0.531510 & \text { ca } & -0.400318 \\ 8 & -6.630003 & 6.507494 & -2.815811 & \text { os } & -0.324624 \\ 8 & -6.626541 & 8.303330 & 1.775479 & \text { os } & -0.324624 \\ 1 & 7.803869 & -7.698111 & -0.633435 & \text { ha } & 0.165138 \\ 1 & 7.803869 & 7.698111 & -0.633435 & \text { ha } & 0.165138 \\ 1 & -7.803869 & -7.698111 & -0.633435 & \text { ha } & 0.165138 \\ 1 & -7.803869 & 7.698111 & -0.633435 & \text { ha } & 0.165138 \\ 6 & 8.007331 & 6.847990 & -3.017044 & \text { c3 } & -0.048949 \\ 6 & 8.005219 & 8.686267 & 1.696806 & \text { c3 } & -0.048949 \\ 6 & 8.005219 & -8.686267 & 1.696806 & \text { c3 } & -0.048949 \\ 6 & 8.007331 & -6.847990 & -3.017044 & \text { c3 } & -0.048949 \\ 6 & -8.007331 & 6.847990 & -3.017044 & \text { c3 } & -0.048949 \\ 6 & -8.005219 & 8.686267 & 1.696806 & \text { c3 } & -0.048949 \\ 6 & -8.005219 & -8.686267 & 1.696806 & \text { c3 } & -0.048949 \\ 6 & -8.007331 & -6.847990 & -3.017044 & \text { c3 } & -0.048949 \\ 1 & 8.251352 & 6.524278 & -4.038420 & \text { h1 } & 0.084327 \\ 1 & 8.168516 & 7.938731 & -2.931026 & \text { h1 } & 0.084327 \\ 1 & 8.661903 & 6.318400 & -2.299679 & \text { h1 } & 0.084327 \\ 1 & 8.247225 & 9.140043 & 2.667851 & \text { h1 } & 0.084327 \\ 1 & 8.657783 & 7.808699 & 1.530790 & \text { h1 } & 0.084327 \\ 1 & 8.170964 & 9.429365 & 0.894614 & \text { h1 } & 0.084327 \\ 1 & 8.247225 & -9.140043 & 2.667851 & \text { h1 } & 0.084327 \\ 1 & 8.170964 & -9.429365 & 0.894614 & \text { h1 } & 0.084327 \\ 1 & 8.657783 & -7.808699 & 1.530790 & \text { h1 } & 0.084327 \\ 1 & 8.251352 & -6.524278 & -4.038420 & \text { h1 } & 0.084327 \\ 1 & 8.661903 & -6.318400 & -2.299679 & \text { h1 } & 0.084327 \\ 1 & 8.168516 & -7.938731 & -2.931026 & \text { h1 } & 0.084327 \\ 1 & -8.251352 & 6.524278 & -4.038420 & \text { h1 } & 0.084327 \\ 1 & -8.661903 & 6.318400 & -2.299679 & \text { h1 } & 0.084327 \\ 1 & -8.168516 & 7.938731 & -2.931026 & \text { h1 } & 0.084327 \\ 1 & -8.247225 & 9.140043 & 2.667851 & \text { h1 } & 0.084327 \\ 1 & -8.170964 & 9.429365 & 0.894614 & \text { h1 } & 0.084327 \\ 1 & -8.657783 & 7.808699 & 1.530790 & \text { h1 } & 0.084327 \\ 1 & -8.247225 & -9.140043 & 2.667851 & \text { h1 } & 0.084327 \\ 1 & -8.657783 & -7.808699 & 1.530790 & \text { h1 } & 0.084327 \\ 1 & -8.170964 & -9.429365 & 0.894614 & \text { h1 } & 0.084327 \\ 1 & -8.251352 & -6.524278 & -4.038420 & \text { h1 } & 0.084327 \\ 1 & -8.168516 & -7.938731 & -2.931026 & \text { h1 } & 0.084327 \\ 1 & -8.661903 & -6.318400 & -2.299679 & \text { h1 } & 0.084327\end{array}$




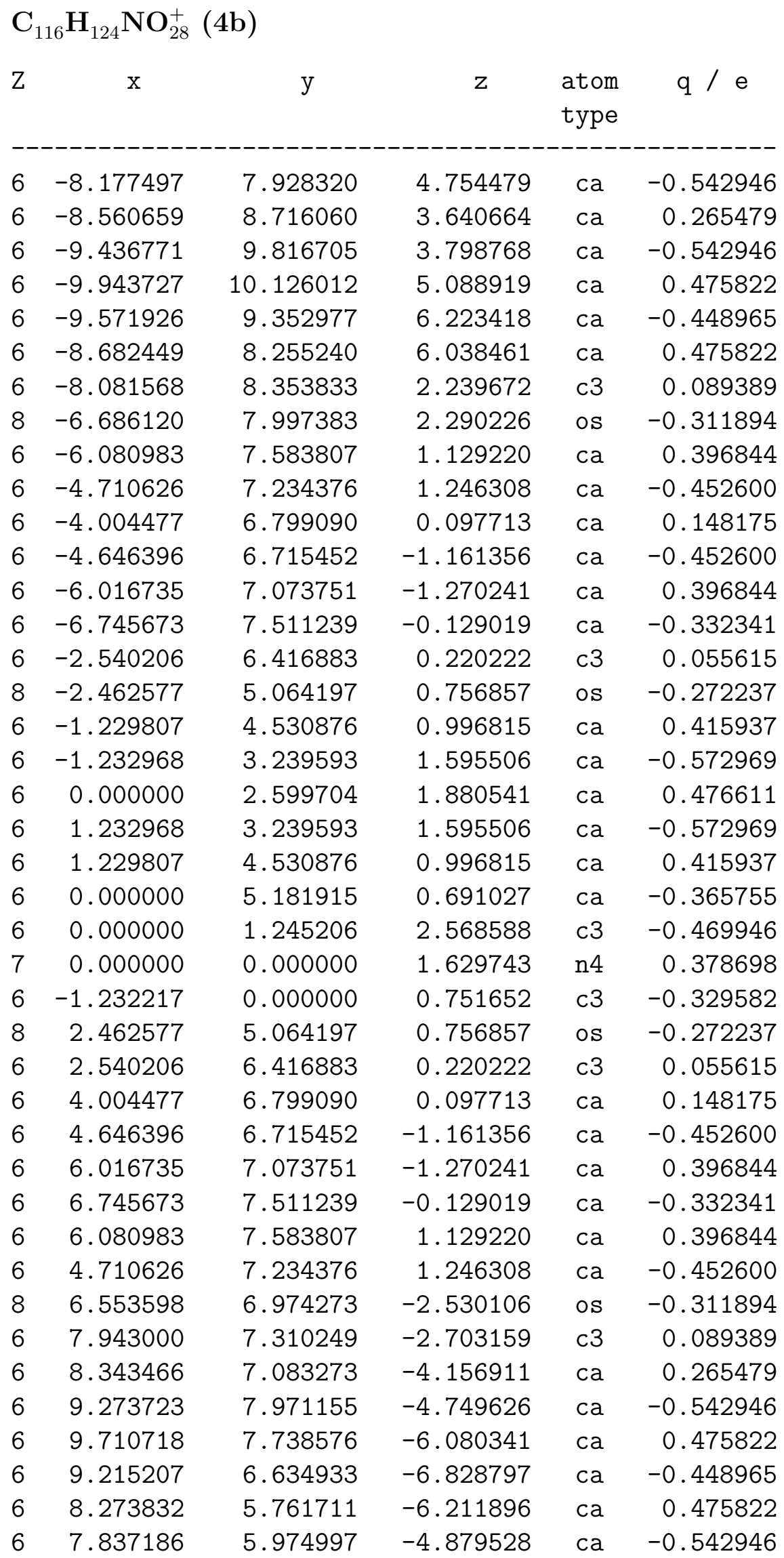




\begin{tabular}{|c|c|c|c|c|c|}
\hline & 6.686120 & 7.997383 & 2.290226 & os & -0 \\
\hline & 8.081568 & 8.353833 & 2.239672 & c3 & 0089389 \\
\hline & 8.560659 & 8.716060 & 3.640664 & $\mathrm{ca}$ & 0.265479 \\
\hline & 8.177497 & 7.928320 & 4.754479 & $\mathrm{ca}$ & -0.542946 \\
\hline & 8.682449 & 8.255240 & 6.038461 & $\mathrm{ca}$ & 0.4758 \\
\hline & 9.571926 & 9.352977 & 6.223418 & $\mathrm{ca}$ & -0.44896 \\
\hline & 9.943727 & 10.126012 & 5.088919 & $\mathrm{ca}$ & 0.47582 \\
\hline & 9.436771 & 9.816705 & 3.798768 & $\mathrm{ca}$ & -0.54294 \\
\hline & -6.553598 & 6.974273 & -2.530106 & os & -0.311894 \\
\hline & -7.943000 & 7.310249 & -2.703159 & c3 & 0.08938 \\
\hline & -8.343466 & 7.083273 & -4.156911 & $\mathrm{ca}$ & 0.265479 \\
\hline & -7.837186 & 5.974997 & -4.879528 & $\mathrm{ca}$ & -0.542946 \\
\hline & -8.273832 & 5.761711 & -6.211896 & $\mathrm{ca}$ & 0.47582 \\
\hline & -9.215207 & 6.634933 & -6.828797 & $\mathrm{ca}$ & -0.448965 \\
\hline & -9.710718 & 7.738576 & -6.080341 & $\mathrm{ca}$ & 0.47582 \\
\hline & -9.273723 & 7.971155 & -4.749626 & $\mathrm{ca}$ & -0.542946 \\
\hline & 10.617855 & 8.650249 & -6.570407 & os & -0.315923 \\
\hline & 11.083333 & 8.483915 & -7.91 & c3 & -0.068158 \\
\hline & 7.726484 & 4.665886 & -6.843656 & os & -0.315923 \\
\hline & 8.123297 & 4.397756 & -8.19 & $c 3$ & -0.068158 \\
\hline & 8.254663 & 7.442502 & 7.06 & os & -0.315923 \\
\hline & 8.729059 & 7.719692 & 878 & c3 & -0.068158 \\
\hline & 10.790708 & 11.2 & 5.14 & OS & -0.315923 \\
\hline & 11.322425 & 11.585197 & 6.4 & c3 & -0.068158 \\
\hline & -7.726484 & 4.665886 & -6.843656 & os & -0.315923 \\
\hline & -8.123297 & 4.397756 & -8.191641 & $c 3$ & -0.068158 \\
\hline & -10.617855 & 8.650249 & $-6.5^{\circ}$ & os & -0.315923 \\
\hline & -11.083333 & 8.483915 & -7.913610 & c3 & -0.068158 \\
\hline & -10.790708 & 11.209265 & 5.144666 & os & -0.315923 \\
\hline & -11.322425 & 11.585197 & 6.419324 & c3 & -0.068158 \\
\hline & -8.254663 & 7.442502 & 7.065726 & os & -0.315923 \\
\hline & -8.729059 & 7.719692 & 8.386878 & $c 3$ & -0.068158 \\
\hline & 1.232217 & 0.000000 & 0.751652 & c3 & -0.329582 \\
\hline & 0.000000 & -1.245206 & 2.568588 & $c$ & -0.46994 \\
\hline & -0.894035 & -1.124016 & 3.196667 & $\mathrm{hx}$ & 0.192447 \\
\hline & 0.000000 & -2.599704 & 1.880541 & $\mathrm{ca}$ & 0.47661 \\
\hline & 1.232968 & -3.239593 & 1.595506 & $\mathrm{ca}$ & -0.57296 \\
\hline & 1.229807 & -4.530876 & 0.996815 & $\mathrm{ca}$ & 0.415937 \\
\hline & 0.000000 & -5.181915 & 0.691027 & $\mathrm{ca}$ & -0.36575 \\
\hline & -1.229807 & -4.530876 & 0.996815 & $\mathrm{ca}$ & 0.415937 \\
\hline & -1.232968 & -3.239593 & 1.595506 & $\mathrm{ca}$ & -0.57296 \\
\hline & 2.462577 & -5.064197 & 0.756857 & os & -0.272237 \\
\hline & 2.540206 & -6.416883 & 0.220222 & c3 & 0.055615 \\
\hline & 4.004477 & -6.799090 & 0.097713 & $\mathrm{ca}$ & 0.14817 \\
\hline & 4.710626 & -7.234376 & 1.246308 & $\mathrm{ca}$ & $-0.4526 c$ \\
\hline
\end{tabular}




\begin{tabular}{|c|c|c|c|c|c|}
\hline & 6.080983 & -7.583807 & 1.129220 & $\mathrm{ca}$ & 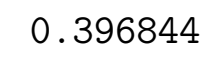 \\
\hline & 6.745673 & -7.511239 & -0.129019 & $\mathrm{ca}$ & -0.332341 \\
\hline & 6.016735 & 7.073751 & -1.270241 & $\mathrm{ca}$ & 0.396844 \\
\hline & 4.646396 & -6.715452 & -1.161356 & $\mathrm{ca}$ & -0.4526 \\
\hline & -2.462577 & -5.064197 & 0.756857 & os & -0.272 \\
\hline & 2.540206 & -6.416883 & 0.220222 & c3 & 0.0556 \\
\hline & -4.004477 & -6.799090 & 0.097713 & $\mathrm{ca}$ & 0.1 \\
\hline & 4.646396 & -6.715452 & -1.161356 & $\mathrm{ca}$ & -0.45 \\
\hline & -6.016735 & -7.073751 & -1.270241 & ca & 0.35 \\
\hline & -6.745673 & -7.511239 & -0.129019 & $\mathrm{ca}$ & -0.33 \\
\hline & 6.080983 & -7.583807 & 1.129220 & $\mathrm{ca}$ & 0.3 \\
\hline & -4.710626 & -7.234376 & 1.246308 & $\mathrm{ca}$ & -0.452 \\
\hline & 6.686120 & -7.997383 & 2.290226 & os & -0.311 \\
\hline & 8.081568 & -8.353833 & 2.23 & $c 3$ & \\
\hline & 8.560659 & -8.716060 & 3.640664 & $\mathrm{ca}$ & 0 . \\
\hline & 9.436771 & -9.816705 & 3.798768 & $\mathrm{Ca}$ & -0 . \\
\hline & 9.943727 & -10.126012 & 5.088919 & $\mathrm{ca}$ & \\
\hline & 9.571926 & -9.352977 & 6.223418 & $\mathrm{ca}$ & -0 . \\
\hline & 8.682449 & 55240 & 6.03 & $\mathrm{ca}$ & \\
\hline & 8.177497 & -7.928320 & 4.754479 & $\mathrm{ca}$ & -0 . \\
\hline & 6.553598 & -6.974273 & -2.53 & os & -0 . \\
\hline & 7.943000 & -7.310249 & -2.70 & c3 & \\
\hline & 8.343466 & -7.083273 & -4.15 & $\mathrm{Ca}$ & 0 \\
\hline & 7.837186 & -5.974997 & -4.87 & $\mathrm{ca}$ & -0 . \\
\hline & 8.273832 & -5.761711 & -6.21 & $\mathrm{ca}$ & 0 . \\
\hline & 9.215207 & -6.634933 & -6.82 & $\mathrm{ca}$ & -0 . \\
\hline & 9.710718 & 38576 & -6.080341 & $\mathrm{ca}$ & 0 . \\
\hline & 9.273723 & -7.971155 & -4.749626 & $\mathrm{Ca}$ & -0 . \\
\hline & -6.553598 & -6.974273 & -2.5 & os & -0 . \\
\hline & -7.943000 & -7.310249 & -2.70 & c3 & \\
\hline & -8.343466 & -7.083273 & -4.15 & $\mathrm{ca}$ & 0 \\
\hline & -9.273723 & -7.971155 & -4.749626 & $\mathrm{ca}$ & -0.542 \\
\hline & -9.710718 & -7.738576 & -6.08 & $\mathrm{ca}$ & 0 \\
\hline & -9.215207 & -6.634933 & -6.82 & $\mathrm{ca}$ & -0.448 \\
\hline & -8.273832 & -5.761711 & -6.211896 & $\mathrm{ca}$ & 0.475 \\
\hline & -7.837186 & -5.974997 & -4.87 & $\mathrm{ca}$ & -0.54294 \\
\hline & -6.686120 & -7.997383 & 2.290226 & os & -0.311 \\
\hline & -8.081568 & -8.353833 & 2.239672 & c3 & 0.089 \\
\hline & -8.560659 & -8.716060 & 3.640664 & $\mathrm{ca}$ & 0.2654 \\
\hline & -8.177497 & -7.928320 & 4.754479 & $\mathrm{ca}$ & -0.542946 \\
\hline & -8.682449 & -8.255240 & 6.038461 & $\mathrm{ca}$ & 0.47582 \\
\hline & -9.571926 & -9.352977 & 6.223418 & $\mathrm{ca}$ & -0.44896 \\
\hline & -9.943727 & -10.126012 & 5.088919 & $\mathrm{ca}$ & 0.47582 \\
\hline & -9.436771 & -9.816705 & 3.798768 & $\mathrm{ca}$ & -0.542 \\
\hline & 7.726484 & -4.665886 & -6.843656 & os & -0.315 \\
\hline
\end{tabular}




\begin{tabular}{|c|c|c|c|c|c|}
\hline & 8.123297 & -4.397756 & -8.191641 & c3 & -0.068158 \\
\hline & 10.617855 & -8.650249 & -6.570407 & os & -0.315923 \\
\hline & 11.083333 & -8.483915 & -7.913610 & c3 & -0.068158 \\
\hline & 10.790708 & 11.209265 & 5.144666 & os & -0.315923 \\
\hline & 11.322425 & -11.585197 & 6.419324 & c3 & -0.068158 \\
\hline & 8.254663 & -7.442502 & 7.065726 & os & -0.31592 \\
\hline & 8.729059 & -7.719692 & 8.386878 & c3 & -0.068158 \\
\hline & -10.617855 & -8.650249 & -6.570407 & os & -0.31592 \\
\hline & -11.083333 & -8.483915 & -7.913610 & c3 & -0.06815 \\
\hline & -7.726484 & -4.665886 & -6.843656 & os & -0.315923 \\
\hline & -8.123297 & -4.397756 & -8.191641 & c3 & -0.068158 \\
\hline & -8.254663 & -7.442502 & 7.065726 & os & -0.315923 \\
\hline & -8.729059 & -7.719692 & 8.386878 & c3 & -0.06815 \\
\hline & -10.790708 & -11.209265 & 5.144666 & os & -0.315923 \\
\hline & -11.322425 & -11.585197 & 6.419324 & c3 & -0.06815 \\
\hline & 1.221693 & 0.903173 & 0.129906 & $\mathrm{hx}$ & 0.153870 \\
\hline & 2.125287 & 0.000000 & 1.392202 & $h x$ & 0.153870 \\
\hline & 1.221693 & -0.903173 & 0.129906 & $\mathrm{hx}$ & 0.153870 \\
\hline & 0.894035 & -1.124016 & 3.196667 & $h x$ & 0.192447 \\
\hline & -.894035 & 1.124016 & 3.196667 & $h x$ & 0.192447 \\
\hline & 0.894035 & 1.124016 & 3.196667 & $h x$ & 0.192447 \\
\hline & -2.125287 & 0.000000 & 1.392202 & $h x$ & 0.153870 \\
\hline & -1.221693 & 0.903173 & 0.129906 & $h x$ & 0.153870 \\
\hline & -1.221693 & -0.903173 & 0.129906 & $h x$ & 0.153870 \\
\hline & 2.194125 & -2.786239 & 1.848339 & ha & 0.202071 \\
\hline & 0.000000 & -6.168729 & 0.236238 & ha & 0.109284 \\
\hline & -2.194125 & -2.786239 & 1.848339 & ha & 0.202071 \\
\hline & -2.194125 & 2.786239 & 1.848339 & ha & 0.202071 \\
\hline & 0.000000 & 6.168729 & 0.236238 & ha & 0.10928 \\
\hline & 2.1 & 2.786239 & 1.848339 & ha & 0.20207 \\
\hline & 2.009564 & -7.098862 & 0.908526 & h1 & 0.083265 \\
\hline & 2.046786 & -6.441444 & -0.767281 & h1 & 0.08326 \\
\hline & -2.046786 & -6.441444 & -0.767281 & h1 & 0.083265 \\
\hline & -2.009564 & -7.098862 & 0.908526 & h1 & 0.08326 \\
\hline & 2.046786 & 6.441444 & -0.767281 & h1 & 0.083265 \\
\hline & 2.009564 & 7.098862 & 0.908526 & h1 & 0.083265 \\
\hline & -2.009564 & 7.098862 & 0.908526 & h1 & 0.08326 \\
\hline & -2.046786 & 6.441444 & -0.767281 & h1 & 0.083265 \\
\hline & 4.226305 & -7.308947 & 2.222295 & ha & 0.19906 \\
\hline & 7.790462 & -7.796291 & -0.217780 & ha & 0.078404 \\
\hline & 4.112665 & -6.387274 & -2.055954 & ha & 0.19906 \\
\hline & -4.112665 & -6.387274 & -2.055954 & ha & 0.19906 \\
\hline & -7.790462 & -7.796291 & -0.217780 & ha & 0.07840 \\
\hline & -4.226305 & -7.308947 & 2.222295 & ha & 0.19906 \\
\hline & 4.112665 & 6.387274 & -2.055954 & ha & 0.19906 \\
\hline
\end{tabular}




\begin{tabular}{|c|c|c|c|c|c|}
\hline & 7.790462 & 7.796291 & -0.217780 & ha & 0.078404 \\
\hline & 4.226305 & 7.308947 & 2.222295 & ha & 0.199064 \\
\hline & -4.226305 & 7.308947 & 2.222295 & ha & 0.199064 \\
\hline & -7.790462 & 7.796291 & -0.217780 & 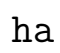 & 0.078404 \\
\hline & -4.112665 & 6.387274 & -2.055954 & & 0.199064 \\
\hline & -8.114825 & -8.365974 & -2.423616 & 1 & 0.073325 \\
\hline & -8.553541 & -6.669840 & -2.035675 & 1 & 0.073325 \\
\hline & -8.658411 & -7.491452 & 1.850465 & 1 & 0.073325 \\
\hline & -8.228833 & -9.209055 & 1.554953 & & 0.073325 \\
\hline & 8.228833 & -9.209055 & 1.554953 & & 0.073325 \\
\hline & 8.658411 & -7.491452 & 1.850465 & & 0.073325 \\
\hline & 8.553541 & -6.669840 & -2.035675 & & 0.073325 \\
\hline & 8.114825 & -8.365974 & -2.423616 & 1 & 0.073325 \\
\hline & 8.114825 & 8.365974 & -2.423616 & - & 0.073325 \\
\hline & 8.553541 & 6.669840 & -2.035675 & 1 & 0.073325 \\
\hline & 8.658411 & 7.491452 & 1.850465 & 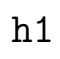 & 0.073325 \\
\hline & 8.228833 & 9.209055 & 1.554953 & 1 & 0.073325 \\
\hline & -8.228833 & 9.209055 & 1.554953 & & 0.073325 \\
\hline & -8.658411 & 7.491452 & 1.850 & & 0. \\
\hline & -8.553541 & 6.669840 & -2.035675 & 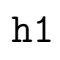 & 0.073325 \\
\hline & -8.114825 & 8.365974 & -2.423616 & 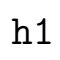 & 0.073325 \\
\hline & 7.110528 & -5.292519 & -4.437828 & a & 0.198615 \\
\hline & 9.545512 & -6.464490 & -7.850614 & & 0.175602 \\
\hline & 9.664502 & -8.837678 & -4.211088 & & 0.198615 \\
\hline & 9.732923 & -10.441319 & 2.952862 & & 0.198615 \\
\hline & 9.95 & -9.5 & 7.210953 & & \\
\hline & 7.492749 & -7.086280 & 4.646736 & & 0.198615 \\
\hline & -9.664502 & -8.837678 & -4.211088 & & 0.198615 \\
\hline & -9.545512 & -6.464490 & -7.850614 & a & 0.175602 \\
\hline & -7.110528 & -5.292519 & -4.437828 & & 0.198615 \\
\hline & -7.492749 & -7.086280 & 4.646736 & la & 0.198615 \\
\hline & -9.956057 & -9.596958 & 7.210953 & & 0.175602 \\
\hline & -9.732923 & -10.441319 & 2.952862 & & 0.198615 \\
\hline & 9.664502 & 8.837678 & -4.211088 & 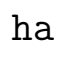 & 0.198615 \\
\hline & 9.545512 & 6.464490 & -7.850614 & & 0.175602 \\
\hline & 7.110528 & 5.292519 & -4.437828 & 110 & 0.198615 \\
\hline & 7.492749 & 7.086280 & 4.646736 & & 0.198615 \\
\hline & 9.956057 & 9.596958 & 7.210953 & & 0.175602 \\
\hline & 9.732923 & 10.441319 & 2.952862 & & 0.198615 \\
\hline & -7.110528 & 5.292519 & -4.437828 & & 0.198615 \\
\hline & -9.545512 & 6.464490 & -7.850614 & 11 & 0.175602 \\
\hline & -9.664502 & 8.837678 & -4.211088 & & 0.198615 \\
\hline & -9.732923 & 10.441319 & 2.952862 & & 0.198615 \\
\hline & -9.956057 & 9.596958 & 7.210953 & & 0.17560 \\
\hline & -7.492749 & 7.086280 & 4.646736 & & 0.198 \\
\hline
\end{tabular}




\begin{tabular}{|c|c|c|c|c|c|}
\hline & 11.959182 & 12.461729 & 6.234234 & h1 & 0.085317 \\
\hline & 10.517604 & 11.861264 & 7.126460 & h1 & 0.085317 \\
\hline & 11.935738 & 10.774299 & 6.855629 & h1 & 0.085317 \\
\hline & 8.270350 & 6.961971 & 9.038037 & h1 & 0.085317 \\
\hline & 9.830431 & 7.633334 & 8.447752 & & .085317 \\
\hline & 8.418890 & 8.726446 & 8.724963 & 1 & 0.085317 \\
\hline & -11.959182 & 12.461729 & 6.234234 & 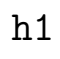 & 0.085317 \\
\hline & -11.935738 & 10.774299 & 6.855629 & + & 0.085317 \\
\hline & -10.517604 & 11.861264 & 7.126460 & 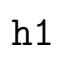 & 0.085317 \\
\hline & -8.270350 & 6.961971 & 9.038037 & & 0.085317 \\
\hline & -8.418890 & 8.726446 & 8.724963 & 1 & 0.085317 \\
\hline & -9.830431 & 7.633334 & 8.447752 & 1 & 0.085317 \\
\hline & -11.784513 & 9.310706 & -8.094724 & 11 & 0.085317 \\
\hline & -10.250733 & 8.545604 & -8.639553 & 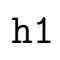 & 0.085317 \\
\hline & -11.613818 & 7.521314 & -8.040542 & 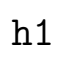 & 0.085317 \\
\hline & -7.573559 & 3.495234 & -8.494859 & 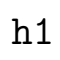 & 0.085317 \\
\hline & -9.210210 & 4.202994 & -8.2 & & 5317 \\
\hline & -7.853741 & 5.232228 & -8.866283 & 1 & 0.085317 \\
\hline & 11.784513 & 9.310706 & -8.09 & - & 0.085317 \\
\hline & 11.613818 & 7.521314 & -8.040542 & h1 & 0.085317 \\
\hline & 10.250733 & 8.545604 & -8.639553 & 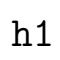 & 0.085317 \\
\hline & 7.573559 & 3.495234 & -8.494859 & 1 & 0.085317 \\
\hline & 7.853741 & 5.232228 & -8.866283 & 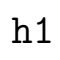 & 0.085317 \\
\hline & 9.210210 & 994 & -8.2 & 1 & 5317 \\
\hline & 8.270350 & -6.961971 & 9.038037 & h1 & 0.085317 \\
\hline & 8.418890 & -8.726446 & 8.724963 & h1 & 0.085317 \\
\hline & 9.830431 & -7.633334 & 8.447752 & h1 & 0.085317 \\
\hline & 11.959182 & -12.461729 & 6.234234 & h1 & 0.085317 \\
\hline & 11.935738 & -10.774299 & 6.855629 & h1 & 0.085317 \\
\hline & 10.517604 & -11.861264 & 7.126460 & h1 & 0.085317 \\
\hline & 11.784513 & -9.310706 & -8.0 & h1 & 0.085317 \\
\hline & 10.250733 & -8.545604 & -8.639553 & h1 & 0.085317 \\
\hline & 11.613818 & -7.521314 & -8.040542 & h1 & 0.085317 \\
\hline & 7.573559 & -3.495234 & -8.494859 & h1 & 0.085317 \\
\hline & 9.210210 & -4.202994 & -8.262589 & h1 & 0.085317 \\
\hline & 7.853741 & -5.232228 & -8.866283 & h1 & 0.085317 \\
\hline & -11.784513 & -9.310706 & -8.094724 & h1 & 0.085317 \\
\hline & -11.613818 & -7.521314 & -8.040542 & h1 & 0.085317 \\
\hline & -10.250733 & -8.545604 & -8.639553 & h1 & 0.085317 \\
\hline & -7.573559 & -3.495234 & -8.494859 & h1 & 0.085317 \\
\hline & -7.853741 & -5.232228 & -8.866283 & h1 & 0.085317 \\
\hline & -9.210210 & -4.202994 & -8.262589 & h1 & 0.085317 \\
\hline & -11.959182 & -12.461729 & 6.234234 & h1 & 0.085317 \\
\hline & -10.517604 & -11.861264 & 7.126460 & h1 & 0.085317 \\
\hline & -11.935738 & -10.774299 & 6.855629 & h1 & 0.085317 \\
\hline
\end{tabular}




$\begin{array}{llllll}1 & -8.270350 & -6.961971 & 9.038037 & \mathrm{~h} 1 & 0.085317 \\ 1 & -9.830431 & -7.633334 & 8.447752 & \mathrm{~h} 1 & 0.085317 \\ 1 & -8.418890 & -8.726446 & 8.724963 & \mathrm{~h} 1 & 0.085317\end{array}$




\section{A.3 AMBER trichloromethane force field}

Table 3: AmBER force field parameters for trichloromethane.

\begin{tabular}{|c|c|c|c|}
\hline involved & \multicolumn{3}{|c|}{ bonding parameters } \\
\hline$\overline{\mathrm{i}-\mathrm{j}}$ & $\begin{array}{r}K_{r} \\
\mathrm{kcal} \mathrm{mol}^{-1}\end{array}$ & $m)^{-2}$ & $\begin{array}{l}r_{\mathrm{eq}} \\
\mathrm{pm}\end{array}$ \\
\hline $\mathrm{c}-\mathrm{h}$ & 340. & & 110.0 \\
\hline $\mathrm{c}-\mathrm{cl}$ & 232. & & 175.8 \\
\hline $\mathrm{i}-\mathrm{j}-\mathrm{k}$ & $\begin{array}{c}K_{\varphi} \\
\text { kcal mol }^{-1}\end{array}$ & $\operatorname{gree}^{-2}$ & $\begin{array}{c}\varphi_{\text {eq }} \\
\text { degree }\end{array}$ \\
\hline $\mathrm{h}-\mathrm{c}-\mathrm{cl}$ & 38.1 & & 107.68 \\
\hline $\mathrm{cl}-\mathrm{c}-\mathrm{cl}$ & 77.7 & & 111.30 \\
\hline atom type & \multicolumn{3}{|c|}{ non-bonding parameters } \\
\hline $\mathrm{i}$ & 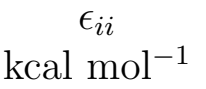 & $\begin{array}{l}\sigma_{i i} \\
\mathrm{pm}\end{array}$ & $\begin{array}{c}q_{\mathrm{RESP}} \\
e\end{array}$ \\
\hline $\mathrm{h}$ & 0.1094 & 190.8 & 0.2659 \\
\hline $\mathrm{c}$ & 0.3250 & 200.0 & -0.3847 \\
\hline $\mathrm{cl}$ & 0.0157 & 118.7 & 0.0396 \\
\hline
\end{tabular}

\section{A.4 Translation diffusion coefficients of trichloromethane}

The translation diffusion coefficients $D$ of trichloromethane in the different dendrizyme solutions were obtained from the trajectories in the NVE ensemble by use of the EinsteinSmoluchowski equation

$$
D=\frac{\left\langle r^{2}\right\rangle}{6 t}
$$

i.e. from the slopes of least-square fits of $\left\langle r^{2}\right\rangle$ (the mean square displacement) of trichloromethane carbon and hydrogen atoms against time. The error estimates in Table 4 are based on the different results obtained for carbon and hydrogen atoms. 
Table 4: Translation diffusion coefficients $D$ of trichloromethane molecules in the examined dendrizyme model systems from trajectories in the $N V E$ ensemble.

\begin{tabular}{|c|r|r|}
\hline $\begin{array}{c}\text { dendrizyme } \\
\text { model system }\end{array}$ & $\begin{array}{c}D \\
10^{-9} \mathrm{~m}^{2} \mathrm{~s}^{-1}\end{array}$ & $\begin{array}{r}\text { regression } \\
\text { coefficient }\end{array}$ \\
\hline \hline $\mathbf{3 g} \mathbf{1 h}$ & $1.57 \pm 0.1$ & 0.999 \\
$\mathbf{3 g 2 h}$ & $1.58 \pm 0.1$ & 0.999 \\
$\mathbf{3 g} 1 \mathbf{w}$ & $1.36 \pm 0.1$ & 0.999 \\
$\mathbf{3 g} \mathbf{2} \mathbf{w}$ & $1.32 \pm 0.1$ & 1.000 \\
$\mathbf{6 g} \mathbf{1 h}$ & $1.23 \pm 0.1$ & 0.999 \\
$\mathbf{6 g} \mathbf{2 h}$ & $1.43 \pm 0.1$ & 0.999 \\
$\mathbf{6 g} \mathbf{1} \mathbf{w}$ & $1.42 \pm 0.1$ & 1.000 \\
$\mathbf{6 g} \mathbf{2} \mathbf{w}$ & $1.45 \pm 0.1$ & 1.000 \\
\hline
\end{tabular}

\section{A.5 Energy conservation in NVE ensemble simulations}

The energy conservation in the NVE ensemble simulations is demonstrated by Figure 1, showing the energy (in $\mathrm{kcal} / \mathrm{mol}$ ) of every saved phased space point for all examined dendrizyme model solutions. No significant energy drifts or discontinuities are visible. 


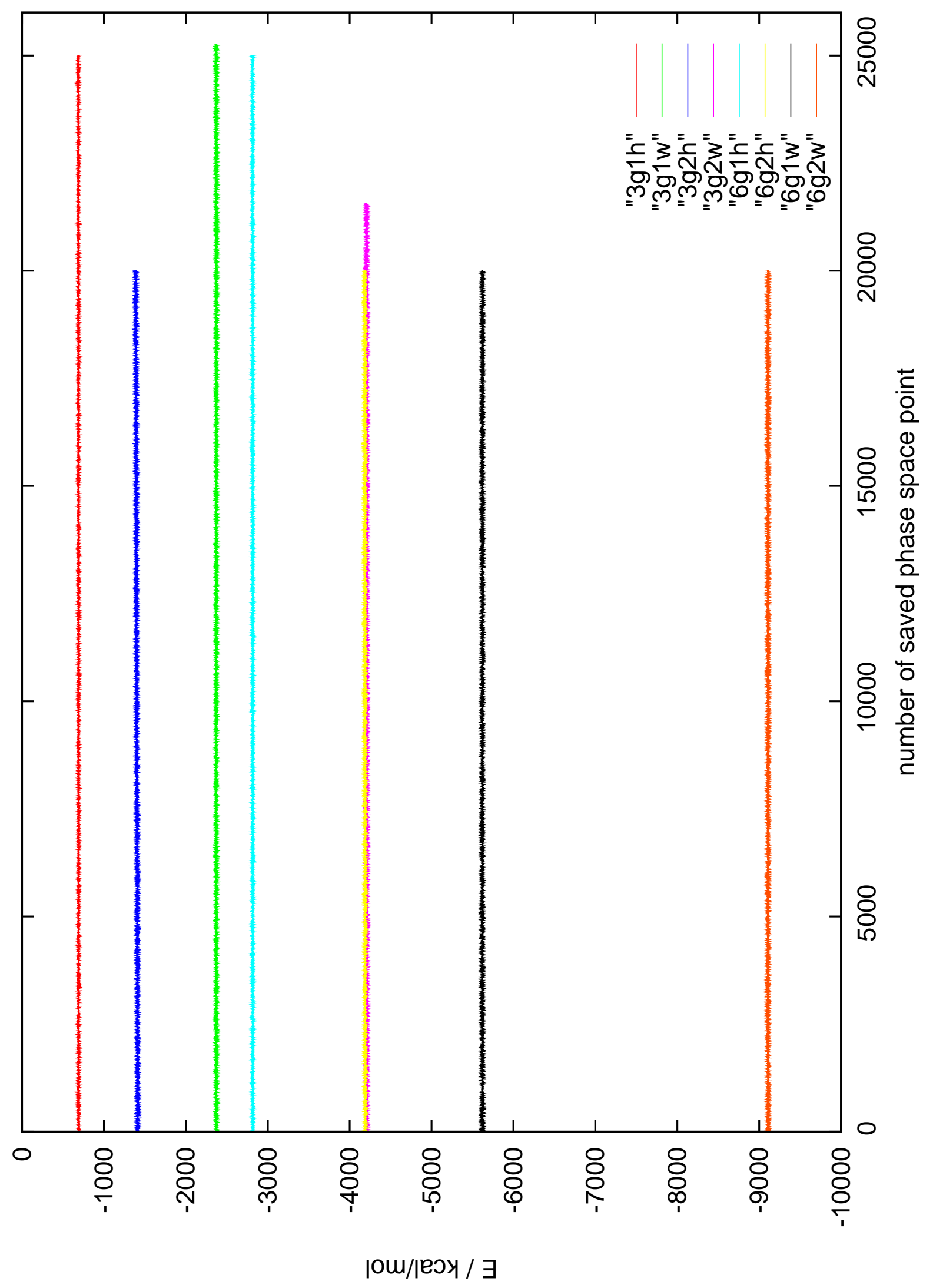

Figure 1: Energy of saved phased space points for all examined dendrizyme model solutions. 


\section{References}

1. Frisch, M. J. et al. Gaussian03, Revision C.02; Gaussian Inc.: Wallingford CT, 2004.

2. Becke, A. D. J. Chem. Phys. 1993, 98, 5648-5652.

3. Lee, C.; Yang, W.; Parr, R. G. Phys. Rev. B: Condens. Matter 1988, 37, 785-789.

4. Stevens, W. J.; Basch, H.; Krauss, M. J. Chem. Phys. 1984, 81, 6026-6033.

5. Stevens, W. J.; Krauss, M.; Basch, H.; Jasien, P. G. Can. J. Chem. 1992, 70, 612-630.

6. Schmidt, M. W.; Baldridge, K. K.; Boatz, J. A.; Elbert, S. T.; Gordon, M. S.; Jensen, J. H.; Koseki, S.; Matsunaga, N.; Nguyen, K. A.; Su, S.; Windus, T. L.; Dupuis, M.; Montgomery, Jr., J. A. J. Comput. Chem. 1993, 14, 1347-1363.

7. Nakano, H. J. Chem. Phys. 1993, 99, 7983-7992.

8. Nakano, H. Chem. Phys. Lett. 1993, 207, 372-378. 